\title{
A Single-Extremity Staged Approach for Critical Congenital Heart Disease Screening: Results from Tennessee
}

\author{
William Walsh ${ }^{1, *}$ and Jean A. Ballweg ${ }^{2}$ \\ 1 Department of Pediatrics, Vanderbilt University Medical Center, Nashville, TN 37232, USA \\ 2 Department of Pediatrics, University of Nebraska Medical Center, Nashville, TN 37232, USA; \\ jballweg@childrensomaha.org \\ * Correspondence: bill.walsh@vandbilt.edu
}

Received: 9 October 2017; Accepted: 14 November 2017; Published: 20 November 2017

\begin{abstract}
Tennessee initiated single-extremity staged screening by pulse oximetry for undetected CCHD in 2012. The algorithm begins with a saturation reading in the foot and allows an automatic pass if the foot pulse oximetry is $97 \%$ or greater. This was based on the principle that it is not possible to have a greater than $4 \%$ difference in the pulse oximetry between upper and lower extremities if the lower extremity is equal to or greater than $97 \%$. This approach eliminates over 75,000 "unnecessary" pulse oximetry determinations in Tennessee each year without affecting the ability to detect CCHD before hospital discharge.
\end{abstract}

Keywords: pulse oximetry screening; screening algorithm; critical congenital heart disease; state screening; coarctation of aorta

\section{Introduction}

Congenital heart disease is present in approximately one in every one hundred live births in the United States. Critical congenital heart disease (CCHD) comprises a significant percentage of heart lesions. The Center for Disease Control (CDC) has identified seven congenital heart lesions that are deemed critical diseases. These seven lesions include hypoplastic left heart syndrome, pulmonary atresia, tetralogy of Fallot, total anomalous venous return, transposition of the great arteries, tricuspid atresia and truncus arteriosus.

Until pulse oximetry screening in the United States was begun, about $30 \%$ of infants with CCHD—or 6 per 10,000 babies—with the seven critical congenital heart disease lesions identified by the CDC were not diagnosed until after initial hospital discharge [1].

With the recommendation for screening in 2011, the USA Secretary of Health and Human Services recommended routine newborn pulse oximetry screening be performed prior to hospital discharge. As of 2015, 46 states and the District of Columbia require hospitals to screen newborns for critical CCHDs [2].

The protocol recommended by the AHA and CDC for screening was based on extensive review of evidence from major European trials [3-6].

In 2011, the AHA and the CDC decided upon a protocol that evaluates the pulse oximetry reading of the infant on a hand and foot after $24 \mathrm{~h}$ of age. To avoid the problem of multiple false positives, it was decided to repeat the screen twice prior to declaring the infant a failure [7].

Seven key lesions which would be expected to have a low saturation were targeted by the AHA and comprise the group of lesions referred to as CCHD as defined above. In addition to the 7 identified CCHDs, the CDC has targeted an additional five lesions when studying CCHD screening. 
These five lesions include coarctation of the aorta, double outlet right ventricle, Ebstein's anomaly, interrupted aortic arch and single ventricle lesions other than HLHS.

This protocol for screening two extremities was based on the possibility that a baby with secondary targets such as coarctation may have decreased saturations in the lower extremity compared to the upper, and therefore a persistent difference greater than 3\% between upper and lower extremity was considered an indication for further evaluation. Using this protocol, it was estimated that about half the infants with CCHD who would have been missed by lack of prenatal diagnosis and absence of signs in the newborn nursery would be identified by a failed pulse oximetry screening test [8].

It was estimated that, each year, about 875 more newborns with a CCHD could be identified at birth hospitals using pulse oximetry newborn screening, but an equal number ( 880 babies) might still be missed each year in the United States. Lesions that were of concern for being missed and significant included truncus arteriosus, coarctation of the aorta and interrupted aortic arch.

The state of Tennessee had been evaluating the possibility of screening since 2006 [9]. We and the state reported on the incidence of missed CCHD, and identified that a diagnostic gap existed in Tennessee prior to the beginning of state-wide screening [10].

Based on our experience and the literature, it was estimated that pulse oximetry would detect CCHD in 5 to 7 Tennessee infants who would otherwise be missed. It was also suggested that the upper extremity pulse oximetry reading would be unnecessary if an initial foot pulse oximetry reading was $97 \%$ or higher, since it would be impossible to have a difference of greater than $3 \%$. Therefore, the Genetics Advisory Committee of the state of Tennessee presented to the Commissioner of Health a modified Tennessee algorithm with an initial assessment of a single lower extremity reading, which, if $97 \%$ or higher a second, upper extremity, test was not required. Figure 1 The two-year results of this screening algorithm are presented in this report.

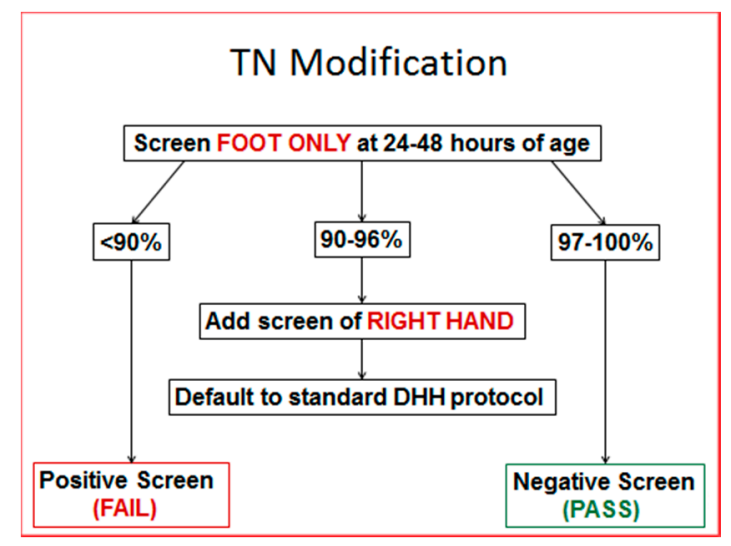

Figure 1. Foot only screen, if the pulse oximetry level is $97 \%$ or higher, the test is a pass and no upper extremity result is obtained, if the level is less than $90 \%$ the infant fails and is referred for further evaluation. A measurement of $90-96 \%$ results in a default to the AHA, CDC algorithm.

\section{Materials and Methods}

The State of Tennessee Health Department collected data on pulse oximetry screening as part of the State Genetics screening program on all live births. The State established a separate CCHD screening task force to monitor and assess newborn pulse oximetry screening. The pulse oximetry data are gathered from the pulse oximetry screening form on each metabolic screening blood spot test.

Surveillance for missed cases of CCHD was done through reports from the separate State of Tennessee Birth Defects Registry and the TIPQC missed-CCHD database as previously reported [10].

De-identified information collected by the TIPQC registry of missed cases included the neonate's diagnosis, age at diagnosis, presenting symptoms, and outcome. For the purpose of the registry, CCHD was defined as severe and life-threatening CHD requiring either surgical or catheter-based 
intervention in the first month of life. Reportable lesions included the 12 CDC targeted lesion which were ductal dependent lesions and lesions resulting in hypoxia. Acyanotic and non-ductal-dependent congenital heart defects requiring semi-elective surgical repair (tetralogy of Fallot without cyanosis, atrioventricular septal defect, atrial septal defect, ventricular septal defect, and patent ductus arteriosus) were excluded from the study.

\section{Results}

In 2013, there were 84,964 births, and in 2014 there were 87,184 births, for a total of 172,148 births in 2013-2014. During that time 95\% $(163,699)$ submitted pulse oximetry screening data. From that cohort, $96 \%$ passed with foot only evaluations. This eliminated the need for a second pulse oximetry reading in 156,948 infants.

For these 2 years, 232 infants failed the screen, with 51 true positives, or $22 \%$. Eight babies had no prenatal suspicion or clinical signs, and were picked up solely by the screening tool. During the same period, there were 13 infants with CCHD not picked up by the screen. Ten of these had left-sided obstruction, which subsequently presented with clinical signs between day of life 3 and 30 . Two infants were found to have TAPVR, one of whom was found on autopsy review after unexplained death at 14 days of age. One baby with no recorded screen presented with Tetralogy of Fallot and one with coactation presented at 9 days of age.

\section{Discussion}

Pulse oximetry screening for CCHD is a valuable adjunct to physical exam and clearly worth the effort. Even in the false positive cases, there are a significant number of infants with other disorders who benefit from evaluation and treatment. We did not systematically track all the babies who failed and who did not have CCHD, but we found a case of intracranial hemorrhage with apnea, a nasal lacrimal tumor, several babies with pneumonia and sepsis. It is clear that no baby should be discharged with a saturation of less than $95 \%$ without a clinical diagnosis [11].

The Tennessee algorithm saved 150,000 unnecessary pulse oximetry readings and still detected the predicted number of CCHD cases.

Using the cost data from Peterson et al. [12], and assuming the pulse oximeter probe is not changed between sites, the time necessary for screening would be reduced from 9 to $5 \mathrm{~min}$; based on the Peterson approximation of average hourly nursing salary, the labor cost would be reduced from $\$ 6.68$ to $\$ 3.71$ per screen; it would therefore cost approximately $\$ 3.00$ per baby, or over $\$ 240,000$ per year, in Tennessee to obtain a pulse oximetry reading in the upper extremity, which could not be more than $3 \%$ greater than the foot reading. In addition to this financial cost, nursing time is valuable and better-used counseling families on newborn care, including Safe Sleep teaching or feeding.

In discussion with physician heath care leaders in other states, the major objection to using the Tennessee approach is the lack of endorsement by the CDC, and the concern that it is "too complicated". However, in discussion with nursing leaders, it is almost instantaneously understood that if the foot saturation is $97 \%$ or higher, it is not possible for the hand to be over $3 \%$ higher, and thus an upper extremity saturation is unnecessary. Thangaratinam et al., in their meta-analysis [13], actually showed no benefit from additional two-extremity testing. However, it is clear from the data reported that, although they are rare, babies with a true differences in saturation due to right to left ductal shunting can be identified, and the two-extremity test should be used whenever there is a possibility of an abnormal result. In the future, new technology may also allow two-site pulse oximetry to detect coarctation of the aorta by using pulsatility information [14].

The possibility of a reversal of saturations due to a TGA is not uncommon, but this condition would also have to be associated with a saturation of over $97 \%$ in the foot. To date, there have been no actual reports of such a condition. To require an additional 150,000 pulse oximetry readings each year looking for such a rare condition would not be cost-effective. 
The 13 babies over two years with false negative results are particularly concerning. It is difficult to identify false negative cases, and we were fortunate in developing a state-wide voluntary reporting system. No screen result was found on 2 infants, one baby with Tetralogy had no screen recorded, and was found to be a home birth without pulse oximetry available. The other baby with coarctation presented at 9 days, and had no screen reported; this was during the first month of establishing the screening program. We have subsequently worked with our mid-wives in $\mathrm{TN}$, and all have portable pulse oximetry capability and education. In addition, through the state genetics quality improvement program, there is now documented screening on over $97 \%$ of all infants. Tennessee does not require the actual saturation levels to be reported, just pass or fail, and whether one or two extremities were tested. The 11 false negatives that were screened passed with a single lower extremity test of over $97 \%$. Nine of these infants were left-sided obstructed lesions, and upon final presentation had saturations ranging from 92 to 100\%. There was one TAPVR who presented with respiratory distress, and there was one death of a baby with TAPVR who presented at 2 weeks of age in arrest; both had passed the screen. Without prospective saturation data, it is not possible to know whether some of these babies would have been diagnosed by the CDC algorithm.

Extrapolating the savings from using the Tennessee approach nationwide would result in 3.8 million fewer pulse oximetry tests, representing approximately $\$ 10$ million dollars in savings without a loss of screening efficacy [12].

A major drawback to a staged single-extremity initial oximetry testing is the need for reeducation and recreation of the multiple, already existent, excellent educational programs in place in many states. CDC and AHA policy makers may decide that such effort may not be worth the benefit. Certainly, for those states that have not yet established a pulse oximetry screening program, using the most efficient approach would be beneficial.

\section{Conclusions}

The Tennessee single extremity algorithm can more efficiently detect infants with CCHD and should be considered by new screening programs.

Author Contributions: William Walsh Conceived of the single extremity pulse oximetry algorithm, organized the State of Tennessee to use it, created the TIPQC task force and reviewed all the collected data and wrote this report. Jean A. Ballweg Detected and reviewed missed cases, provided feedback to the state CCHD detection committee and reviewed and edited this article.

Conflicts of Interest: The authors declare no conflict of interest.

\section{Abbreviations}

$\begin{array}{ll}\text { AAP } & \text { Academy of Pediatrics } \\ \text { AHA } & \text { American Heart Association } \\ \text { CHD } & \text { Congenital heart disease } \\ \text { CCHD } & \text { Critical congenital heart disease } \\ \text { CI } & \text { Confidence interval } \\ \text { COA } & \text { Coarctation of the aorta } \\ \text { TIPQC } & \text { Tennessee Initiative for Perinatal Quality Care } \\ \text { TN } & \text { Tennessee }\end{array}$

\section{References}

1. Wren, C.; Reinhardt, Z.; Khawaja, K. Twenty-year trends in diagnosis of life-threatening neonatal cardiovascular malformations. Arch. Dis. Child. 2008, 93, F33-F35. [CrossRef] [PubMed]

2. Oster, M.E.; Aucott, S.W.; Gildewell, J.; Hackell, J.; Kohilas, L.; Martin, G.R.; Phillippi, J.; Pinto, N.M.; Saarinen, A.; Sontag, M.; et al. Lessons Learned From Newborn Screening for Critical Congenital Heart Defects. Pediatrics 2016, 137, e20154573. [CrossRef] [PubMed] 
3. Riede, F.T.; Worner, C.; Dahnert, I.; Mockel, A.; Kostelka, M.; Schneider, P. Effectiveness of neonatal pulse oximetry screening for detection of critical congenital heart disease in daily clinical routine-Results from a prospective multicenter study. Eur. J. Pediatr. 2010, 169, 975-981. [CrossRef] [PubMed]

4. De-Wahl Granelli, A.; Wennergren, M.; Sandberg, K.; Mellander, M.; Bejum, C.; Inganas, L.; Eriksson, M.; Segerdahl, N.; Ågren, A.; Ekman-Joelsson, B.-M.; et al. Impact of pulse oximetry screening on the detection of duct dependent congenital heart disease: A Swedish prospective screening study in 39,821 newborns. BMJ 2009, 338, a3037. [CrossRef] [PubMed]

5. $\quad$ Ewer, A.K.; Middleton, L.J.; Furmston, A.T.; Bhoyar, A.; Daniels, J.P.; Thangaratinam, S.; Deeks, J.J.; Khan, K.S.; PulseOx Study Group. Pulse oximetry screening for congenital heart defects in newborn infants (PulseOx): A test accuracy study. Lancet 2011, 378, 785-794. [CrossRef]

6. Thangaratinam, S.; Daniels, J.; Ewer, A.K.; Zamora, J.; Khan, K.S. Accuracy of pulse oximetry in screening for congenital heart disease in asymptomatic newborns: A systematic review. Arch. Dis. Child. 2007, 92, F176-F180. [CrossRef] [PubMed]

7. Kemper, A.R.; Mahle, W.T.; Martin, G.R.; Cooley, W.C.; Kumar, P.; Morrow, W.R.; Kelm, K.; Pearson, G.D.; Glidewell, J.; Grosse, S.D.; et al. Strategies for Implementing Screening for Critical Congenital Heart Disease. Pediatrics 2011, 128, e1259-e1267. [CrossRef] [PubMed]

8. Ailes, E.C.; Gilboa, S.M.; Honein, M.A.; Oster, M.E. Estimated Number of Infants Detected and Missed by Critical Congenital Heart Defect Screening. Pediatrics 2015, 135, 1001-1012. [CrossRef] [PubMed]

9. Liske, M.R.; Greeley, C.S.; Law, D.J.; Reich, J.D.; Morrow, W.R.; Baldwin, H.S.; Graham, T.P.; Strauss, A.W.; Kavanaugh-McHugh, A.L.; Walsh, W.F. Report of the Tennessee Task Force on Screening Newborn Infants for Critical Congenital Heart Disease. Pediatrics 2006, 118, 1250-1256. [CrossRef] [PubMed]

10. Mouledoux, J.H.; Walsh, W.F. Evaluating the Diagnostic Gap: Statewide Incidence of Undiagnosed Critical Congenital Heart Disease before Newborn Screening with Pulse Oximetry. Pediatr. Cardiol. 2013, 34, 1680-1686. [CrossRef] [PubMed]

11. Ewer, A.K.; Furnston, A.T.; Middleton, L.J.; Deeks, J.J.; Daniels, J.P.; Pattison, H.M.; Powell, R.; Roberts, T.E.; Barton, P.; Auguste, P.; et al. Pulse oximetry as a screening test for congenital heart defects in newborn infants: A test accuracy study with evaluation of acceptability and cost-effectiveness. Health Technol. Assess. 2012, 16, 1-184.

12. Peterson, C.; Grosse, S.D.; Oster, M.E.; Olney, R.S.; Cassell, C.H. Cost-effectiveness of routine screening for critical congenital heart disease in US newborns. Pediatrics 2013, 132, e595-e603. [CrossRef] [PubMed]

13. Thangaratinam, S.; Brown, K.; Zamora, J.; Khan, K.S.; Ewer, A.K. Pulse oximetry screening for critical congenital heart defects in asymptomatic newborn babies: A systematic review and meta-analysis. Lancet 2012, 379, 2459-2464. [CrossRef]

14. Granelli, A.W.; Ostman-Smith, I. Noninvasive peripheral perfusion index as a possible tool for screening for critical left heart obstruction. Acta Paediatr. 2007, 96, 1455-1459. [CrossRef] [PubMed] 